\title{
Successful pregnancy outcome in a case of idiopathic primary hypoparathyroidism with seizure disorder: a case report
}

\author{
Sarita Rajbhar, Sagarika Majumdar*, Pragati Trigunait, Pushpawati Thakur, \\ Taru Shikha, Aparajita Rastogi
}

Department of Obstetrics and Gynaecology, AIIMS Raipur, Chhattisgarh, India

Received: 28 November 2020

Accepted: 24 December 2020

\section{*Correspondence:}

Dr. Sagarika Majumdar,

E-mail: sagarikamajumdar84@gmail.com

Copyright: (c) the author(s), publisher and licensee Medip Academy. This is an open-access article distributed under the terms of the Creative Commons Attribution Non-Commercial License, which permits unrestricted non-commercial use, distribution, and reproduction in any medium, provided the original work is properly cited.

\begin{abstract}
Hypoparathyroidism, a disorder of low parathyroid hormones resulting in hypocalcemia and hyperphosphatemia, is relatively a rare occurrence in pregnancy. Nevertheless, it has been reported to cause significant maternal and neonatal morbidity and even mortality in severe cases. Dynamic changes in serum calcium during pregnancy superimposed with the inherent defect in the parathyroid gland and calcium metabolism in these patients make it even more difficult to monitor and manage the patient. We report a case of idiopathic primary hypoparathyroidism with seizure disorder, managed with aggressive monitoring and timely decision making, resulting in a favorable outcome.
\end{abstract}

Keywords: Primary hypoparathyroidism, Hypocalcaemia, Pregnancy

\section{INTRODUCTION}

Hypoparathyroidism is an orphan endocrine disorder characterized by hypocalcemia, absence or inappropriately low circulating PTH levels and normal to high phosphate level with varying etiologies. ${ }^{1}$ Hypoparathyroidism in pregnancy is seldom encountered by obstetricians. The prevalence of hypoparathyroidism ranges from 0.5 to $6.6 \%$ worldwide, however there is no available data in pregnant women. ${ }^{2}$ Hypoparathyroidism can be of primary or secondary type. Primary hypoparathyroidism (PHP) results from absence or deficient production of parathyroid hormone (PTH). Causes most commonly includes iatrogenic events like surgical injury/inadvertent removal of parathyroid gland during thyroid/parathyroid surgery, followed by noniatrogenic causes like autoimmune destruction of the parathyroid gland as seen in autoimmune polyglandular syndrome type 1, genetic abnormalities (Di Georges syndrome) or other acquired causes like radiotherapy or infiltrative diseases (Wilson's disease ,granulomas or metastatic disease). ${ }^{2,3}$ However, specific causes cannot be identified promptly in few cases(Idiopathic). In Secondary hypoparathyroidism, parathyroid gland dysfunction is the consequence of a primary problem elsewhere, leading to hypercalcinemia finally resulting in low PTH levels. ${ }^{4}$

PHP in pregnancy has been seen to be associated with maternal morbidity and fetal loss. ${ }^{2,5,6}$ In pregnancy with hypoparathyroidism, physiological rise in calcitriol level during pregnancy enhances the intestinal calcium absorption which in turn further reduce the PTH level leading to hypercalciuria. This sometimes increases the risk of renal stones during pregnancy. In untreated PHP, hypocalcemia in fetus stimulates fetal parathyroid gland to secrete excess PTH hormone subsequently causing fetal hyperparathyroidism and demineralization of fetal skeleton with severe cases presenting with low birth weight, preterm labor, recurrent spontaneous abortion and possibly intrauterine fetal demise. Similarly overzealous treatment of the disease with calcium and vitamin D, a recommended medical management for the disease, may cause hypercalcinemia, which in turn suppresses fetal 
parathyroid gland resulting in hypocalcemia in newborn. ${ }^{6,7}$ Hence it is important to closely monitor and maintain an optimal serum calcium (preferably in a low normal reference range) and PTH level both during pregnancy and lactation. ${ }^{6}$

The clinical course and therapeutic management of hypoparathyroidism in pregnancy is still controversial, due to the lack of adequate case reports or case series published in literature.

We herein report a case of pregnant patient with primary hypoparathyroidism with seizure disorder culminating in successful pregnancy outcome.

\section{CASE REPORT}

A 35-year-old female, gravida 2, para 1, living 1 at 38 weeks period of gestation with previous LSCS (lower segment caesarean section), reported to our OPD with complaints of pain abdomen for 1 day. She was a known case of primary hypoparathyroidism with seizure disorder diagnosed 3 years back in a tertiary care hospital. She was admitted under our care for further management. On eliciting history carefully, she recalled that she had her first seizure episode when she was 6 years old. It was a GTCS (generalised tonic clonic type of seizure) with uprolling of eyes and frothing from mouth. She was asymptomatic thereafter. However, she didn't give any history of headache, vomiting and tongue bite or any incontinence. Her second seizure episode was on postoperative day 3 of emergency LSCS (lower segment c-section) which was done in view of PROM (pre-labor rupture of membrane) with non-progress of labour in 2005 when she had history of tongue bite with post-ictal state, and history similar to carpopedal spasm. However, her case remained undiagnosed at that time and no specific treatment was given to her. The baby delivered was healthy but had one episode of seizure on day 2 of her life, there was no biochemical or structural abnormality detected in the child. In 2017 , she went to a tertiary care hospital for further evaluation and treatment under department of neurology as the frequency of seizure episode rose suddenly along with increased intensity of myalgia, polyarthralgia, lethargy and tingling of feet over 2 year. Simultaneously, MRI and CT scan brain with contrast showed no abnormality and EEG done was normal as well. Genetic testing was not done. Her biochemical tests were suggestive of low serum calcium, low serum vitamin D and low PTH levels with elevated phosphate levels. She was transferred to endocrinology department for further evaluation. Symptoms got improved with intravenous calcium gluconate. She was diagnosed as a case of primary hypoparathyroidism with seizure disorder (probably secondary to hypocalcaemia) and was discharged on an OPD basis on tablet calcium, tablet calcitriol and sodium valproate. She did not have any history of tuberculosis, malignancy, vitiligo, alopecia or recurrent candidiasis, head and neck surgery or history of radiation, fever, headache, blurring of vision in the past. Her family history did not reveal similar events or history of any autoimmune disease.

Table 1: Results of laboratory investigations at presentation.

\begin{tabular}{|lll|}
\hline Test & Result & Normal range \\
\hline $\begin{array}{l}\text { Calcium corrected for } \\
\text { albumin (mg/dl) }\end{array}$ & 7.1 & $8.7-10.2$ \\
\hline Ionised calcium (mmol/l) & 1.0 & $1.12-1.32$ \\
\hline Serum Phosphorus (mg/dd) & 5.6 & $3.1-4.8$ \\
\hline Serum Magnesium (mg/dl) & 1.9 & $1.8-2.4$ \\
\hline Serum intact PTH (pg/ml) & 1.7 & $15-65$ \\
\hline Serum albumin (gm/dl) & 3.16 & $4-5$ \\
\hline Serum vitamin D (ng/ml) & 11.41 & $30-100$ \\
\hline Serum TSH $(\mathbf{m I U} \mathbf{I})$ & 4.154 & $0.55-4.78$ \\
\hline Serum creatinine (mg/dl) & 0.8 & $0.6-1.1$ \\
\hline
\end{tabular}

Her present pregnancy was unknown till 2 months of gestation, in which she kept taking tablet sodium valproate. She had her routine antenatal check up in a government hospital near her home where she was advised to stop tablet valproate immediately once the pregnancy was confirmed. She was periodically taking telephonic advice from the tertiary care hospital where she was previously diagnosed. She was advised to do weekly serum calcium initially followed by monthly serum calcium for monitoring. Her antenatal period was uneventful on tablet calcium $500 \mathrm{mg}$ twice daily, and tablet calcitriol $0.25 \mathrm{mg}$ twice daily. Her anomaly scan and growth scans were normal. She was referred to our hospital when she complained of pain abdomen with no episode of leaking or bleeding per vaginum at 38 weeks of gestation. On general physical examination, her vitals were stable, trousseau's sign was positive. Per abdomen examination revealed gravid uterus of 36 weeks size, longitudinal lie, cephalic presentation, relaxed, with no scar tenderness and fetal heart rate was present. On per vaginal examination, OS was closed, cervix was soft and uneffaced with head station high up. Her pelvis was found to be borderline and a diagnosis of CPD (cephalopelvic disproportion) was made. On admission there were no symptoms of hypocalcaemia. ECG done was normal. In parallel, biochemical test (serum calcium, phosphate, magnesium, chloride) were sent and revealed low calcium, low serum PTH, high phosphate and normal magnesium level (Table 1) for which endocrinology opinion was taken. She was advised to continue tablet calcitriol $0.25 \mathrm{mg}$ twice daily and tablet calcium $1000 \mathrm{mg}$ twice daily. She was given a stat dose of one $10 \mathrm{ml}$ ampule of 10 percent calcium gluconate in $500 \mathrm{ml} \mathrm{NS}$ infused intravenously over 1 hour. Ophthalmology reference was done for fundoscopy to rule out papilledema. Patient was kept on close monitoring of serum calcium and serum phosphate perioperatively. Decision of elective LSCS (lower segment caesarean section) was taken on account of previous caesarean birth with cephalopelvic disproportion in a known case of PHP with seizure disorder after pre-anaesthetic check-up and she delivered a healthy term alive female baby of $3.5 \mathrm{~kg}$. 
Repeat biochemical tests were sent on the same postoperative day. Repeat calcium level was $6.2 \mathrm{mg} / \mathrm{dl}$, phosphorus was $4.6 \mathrm{mg} / \mathrm{dl}$ and vitamin D3 was 11.4 $\mathrm{ng} / \mathrm{ml}$, for which 4 ampules of intravenous calcium gluconate infusion and a stat dose of injection Arachitol 3 lakh unit intramuscular was administered. There were no episodes of seizure, carpopedal spasm during intraoperative and postoperative period.

Baby delivered had swelling and hyperpigmentation of labia for which it was suspected to be a case of ambiguous genitalia. However, during work up ultrasound abdomen was within normal limits showing uterus and QF-PCR (quantitative fluorescencepolymerase chain reaction) was also within normal limits. Biochemical tests of the baby were also found to be normal. Both the mother and baby were discharged in stable condition.

\section{DISCUSSION}

Pregnancy brings many physiological changes including changes in calcium metabolism. Dynamic changes in calcium homeostasis during pregnancy results in accumulation of around $30 \mathrm{gm}$ of fetal skeletal calcium by term, which helps in mineralization of bones in fetus. ${ }^{5,8}$ PHP in pregnancy, is diagnosed on the basis of characteristic symptoms, detailed and careful history taking and certain biochemical tests. ${ }^{1,9}$ The diagnostic challenge is to differentiate physiological changes, which occurs in maternal calcium metabolism both during pregnancy and lactation, from the underlying pathology. The therapy has to be customized throughout pregnancy, after delivery and during lactation with rigorous monitoring of serum total or ionized calcium level for successful pregnancy outcome.

Literature reveals, there may be drastic variations in levels of parathyroid hormone (PTH) throughout pregnancy ranging from the low normal reference range in the 1st trimester to subsequent rise in the mid normal reference range by the third trimester. It has been seen that PTH is affected by maternal dietary calcium intake as well as maternal vitamin D level. ${ }^{2,6,10}$

Physiological rise in calcitriol level (from kidney and even placenta to some extent ) during first trimester, as shown in many studies, increase the intestinal calcium absorption to meet the need of fetus which in turn suppress PTH in first trimester and subsequently increase the urinary calcium excretion. ${ }^{2,6-8}$ PTHrP (parathyroid hormone related protein ) is the PTH equivalent in fetus which helps in maintaining maternal-fetal calcium gradient along with vitamin D3. ${ }^{7}$ Source of PTHrP is commonly placenta and breast. It is the key stimulator of renal 1-alpha hydroxylase enzyme which begins to rise from first trimester and increase to three-fold by term. The significant rise in PTHrP may increase the conversion of calcidiol to metabolically active form of vitamin D (1,25-hydroxyvitamin D3/calcitriol) but in turn suppress endogenous PTH. ${ }^{11}$

Although many case reports concluded the rise in calcitriol and PTHrP in patients with hypoparathyroidism during pregnancy which may eventually result in reduced calcium and calcitriol dose requirement during treatment in pregnancy, it has also been emphasised in other studies that the calcium and calcitriol requirement increases among many individuals during pregnancy. ${ }^{6}$ It was found that this might be due to variation in production of PTHrP from placenta and breast among different individuals.

Physiologically, immediate postpartum serum calcium levels in neonate falls in response to decreased PTHrP. During lactation, there are a gradual normalization of maternal 1,25-dihydroxyvitamin D level, reduced levels of intact PTH, and increased plasma levels of phosphates and ionized calcium. ${ }^{4}$ These physiological changes superadded with hypoparathyroidism can bring varied changes in serum levels of calcitriol and calcium and this emphasizes the need for real time monitoring of serum levels in both mother and the fetus for better titration of doses of medication. ${ }^{12}$

Hypoparathyroidism during pregnancy may bring on maternal complications resulting from hypocalcemia (neuromuscular hyperexcitability), obstetrical complications (spontaneous abortion, premature delivery, fetal complications due to compensatory hyperparathyroidism with skeletal demineralization, rib fractures, intrauterine growth retardation, premature delivery, fetal or neonatal death and neonatal complications like respiratory distress, poor feeding and hypotonia. ${ }^{11,13}$

Severity of symptoms in both mother and the neonate are influenced by the level and duration of hypocalcemia. It is characterized by neuromuscular hyper excitability like facial muscle spasms, muscle cramps, and paresthesia of the lips, tongue, fingers, and feet in mild cases and may proceed to tetany, seizures, prolonged QT interval, heart failure, cataracts, ectodermal disorders, dental abnormalities and intracranial calcification in severe and chronic hypocalcemia state. Classic physical findings in patients with neuromuscular irritability due to latent tetany are Trousseau's and Chvostek's signs. ${ }^{8}$

The case presented to us was a diagnosed case of idiopathic primary hypoparathyroidism with seizure disorder for 3 years. Although, she had intermittent history of carpopedal spasms, paranesthesia, lethargy, fatigue and seizures since childhood, she remained undiagnosed and untreated. As her MRI and CT scan brain did not show any abnormality, EEG done was normal and blood parameter showed typical findings of PHP, she was labeled as a case of idiopathic primary hypoparathyroidism. 
Biochemical tests in primary hypoparathyroidism reveals low serum levels of total/ionized calcium, low levels of serum intact parathyroid hormone with elevated serum phosphorus and 24-hour urinary calcium and normal serum magnesium and creatinine level. They typically have normal level of 25-hydroxy vitamin D but normal to low 1,25-hydroxyvitamin D concentrations(as PTH is the main stimulator of 1alpha hydroxylase enzyme which converts 25-hydoxyvitaminD to 1,25 hydroxyvitamin D. ${ }^{11}$

Blood parameters of the patients revealed severely low serum calcium levels with low PTH, and high phosphorus levels. Serum magnesium and serum creatinine were within normal range. This is a usual finding observed in primary hypoparathyroidism.

Management of hypoparathyroidism depends on the severity of presentation and the severity of hypocalcemia state. Goal is to maintain serum calcium in low normal reference range to prevent iatrogenic nephrolithiasis. Close monitoring of serum calcium during pregnancy is the key to success in management of hypoparathyroidism in pregnancy. In milder degree with serum calcium level $>7.5 \mathrm{mg} / \mathrm{dl}$, oral calcium and vitamin D supplementation is preferred. Intravenous calcium is used only if symptom is not alleviated with oral calcium. ${ }^{2,5,7}$

In severe hypoparathyroidism cases with acute decrease in calcium levels to $<7.5 \mathrm{mg} / \mathrm{dl}$, the use of intravenous calcium therapy (i.e., one $10 \mathrm{ml}$ ampule of 10 percent calcium gluconate in $50 \mathrm{ml}$ of 5 percent dextrose infused over 10-20 minutes, followed by intravenous infusion of calcium gluconate) is suggested to correct serum calcium level, even if the patient is asymptomatic. Weaning of intravenous calcium is done once the patient is able to take oral calcium.

Conventionally, the standard treatment for primary hypoparathyroidism in pregnancy is oral calcium supplementation with active vitamin D metabolite (calcitriol) and low phosphate diet, because of their proven safety profile in pregnancy. 5,6,8 The main therapeutic challenge during treatment is to maintain optimum calcium levels by adjusting doses. Because our patient was already on calcium and calcitriol for 3 years, there was a high probability of fluctuating level during pregnancy and lactation. Therefore, in our case careful monitoring and dose adjustment of medications accordingly after admission, were given utmost importance. ${ }^{6,13}$

In contrast to prodrugs like cholecalciferol, calcitriol has a much shorter, dose-independent half-life and hence amenable to better titration of dose in view of hypercalcemia due to overdosing. ${ }^{8}$ Akhtar et al summarized that the risk of vitamin D overdosage and subsequent teratogenicity in humans and animals seems to be small as long as the serum calcium and the 1,25 $(\mathrm{OH}) 2$ D3 concentrations remain in lower range. To keep the serum calcium concentration within normal range (between $8 \mathrm{mg} / \mathrm{dl}$ to $8.5 \mathrm{mg} / \mathrm{dl}$ ), a calcium supplementation of $1 \mathrm{gm} /$ day and calcitriol dose ranging between $0.25 \mu \mathrm{g} /$ day and $3.00 \mu \mathrm{g} /$ day is generally required. ${ }^{5}$

Advanced therapy with PTH replacement (recombinant human PTH) is an attractive option but its safety in pregnancy is still not well evaluated due to paucity of studies. 2,6,13 There is limited data regarding ideal mode of delivery but various case reports concur that caesarean section is decided on the basis of obstetrical indications. ${ }^{7}$

In our patient, we administered intravenous infusion of calcium gluconate (one $10 \mathrm{ml}$ ampule of 10 percent calcium gluconate in $500 \mathrm{ml} \mathrm{NS}$ ) over 1 hour after confirming low calcium values. She was continued on tablet calcium $1 \mathrm{gm}$ twice daily and tablet calcitriol 0.25 mg twice daily. Careful monitoring of serum total calcium and phosphate level was done twice daily. We did an elective Caesarean section, for obstetrical indication as she was a case of previous caesarean birth with cephalopelvic disproportion, which resulted in a successful pregnancy outcome with a healthy alive baby. Post operatively repeat dose of calcium gluconate infusion was given to optimize the serum calcium level. Once she started taking normal diet, she was again switched to oral calcium and vitamin D supplements.

Follow up during pregnancy with serum calcium every 34 weekly is of utmost important to optimize calcium levels and readjust the dose of calcium and calcitriol which helps in preventing hyper or hypocalcemia and reduces significant post-operative morbidity associated with it. Frequent readjustment in dose should be avoided both during pregnancy and lactation, as serum calcium levels also depends on dietary calcium intake and lifestyle factors. $., 7,11,13,14$

After discharge from our hospital, she was in constant follow up with her endocrinologist and close monitoring of serum calcium and phosphate was done once weekly till next 4 weeks. She continued same dose of calcium and calcitriol during lactation as her calcium level was within acceptable limits. Sodium valproate was not restarted.

\section{CONCLUSION}

The obstetrician's approach of a pregnant patient with primary hypoparathyroidism underlines the importance of high level of clinical suspicion because of rarity of such cases. The management of these cases requires a timely and multidisciplinary decision making by a team approach among the obstetricians, neonatologists, anesthetists and endocrinologist for better pregnancy outcome with minimal risk to both mother and the baby. The importance of rigorous monitoring of biochemical parameters is also an absolute necessity throughout pregnancy and lactation. This enables appropriate 
titration of medications and ensures delivery of a patient centric management.

Funding: No funding sources Conflict of interest: None declared

Ethical approval: Not required

\section{REFERENCES}

1. Marques Mendes E, Meireles-Brandão L, Meira C, Morais N, Ribeiro C, Guerra D. Primary hypoparathyroidism presenting as basal ganglia calcification secondary to extreme hypocalcemia. Clin Pract . 2018;8(1):1007.

2. Richa CG, Issa AI, Echtay AS, El Rawas MS. Idiopathic Hypoparathyroidism and Severe Hypocalcemia in Pregnancy. Case Rep Endocrinol. 2018;2018:1-4.

3. Abate EG, Clarke BL. Review of hypoparathyroidism. Frontiers in Endocrinology. Frontiers Media SA. 2017;7:172.

4. Hypoparathyroidism: Practice Essentials, Pathophysiology, Epidemiology. Available from: https://emedicine.medscape.com/article/122207overview. Accessed on 20 Oct, 2020.

5. Akhtar N. Pregnancy with Hypoparathyroidism-A Case Report. Bangabandhu Sheikh Mujib Med Univ J. 2011;3(2):107-9.

6. Khan AA, Clarke B, Rejnmark L, Brandi ML. Management of endocrine disease: Hypoparathyroidism in pregnancy: Review and evidence-based recommendations for management. Eur J Endocrinol. BioScientifica Ltd. 2019;180:R3744.
7. Hatswell BL, Allan CA, Teng J, Wong P, Ebeling PR, Wallace EM, et al. Management of hypoparathyroidism in pregnancy and lactation - A report of 10 cases. Bone Reports. 2015;3:15-9.

8. Bloom S, Corton M, Spong C. Williams Obstetrics, $24^{\text {th }}$ ed. McGraw-Hill. 2014;1376.

9. Kovacs CS. Maternal mineral and bone metabolism during pregnancy, lactation, and post-weaning recovery. Physiol Rev. 2016;96(2):449-547.

10. Mahadevan S, Bharath R, Kumaravel V. Calcium and bone disorders in pregnancy. Indian $\mathrm{J}$ Endocrinol Metab. 2012;16(3):358.

11. Cardot-Bauters C. Hypoparathyroidism and pregnancy. Ann Endocrinol (Paris). 2016;77(2):1725.

12. Al Nozha OM, Malakzadeh-Shirvani P. Calcium homeostasis in a patient with hypoparathyroidism during pregnancy, lactation and menstruation. J Taibah Univ Med Sci. 2013;8(1):50-3.

13. Bollerslev J, Rejnmark L, Marcocci C, Shoback DM, Sitges-Serra A, Van Biesen W, et al. European Society of Endocrinology Clinical Guideline: Treatment of chronic hypoparathyroidism in adults. Eur J Endocrinol. 2015;173(2):G1-20.

14. Alalawi Y, M'Hiri I, Alrob HA, Khan A. Hypoparathyroidism in pregnancy. In: Hypoparathyroidism: A Clinical Casebook. Springer International Publishing. 2019;143-53.

Cite this article as: Rajbhar S, Majumdar S, Trigunait P, Thakur P, Shikha T, Rastogi A. Successful pregnancy outcome in a case of idiopathic primary hypoparathyroidism with seizure disorder: a case report. Int J Reprod Contracept Obstet Gynecol 2021;10:747-51. 